\title{
Nuclear LC3-positive puncta in stressed cells do not represent autophagosomes
}

This letter points out an important potential artifact when using immunolabeling techniques with confocal microscopy to identify autophagosomes. Our laboratory has been investigating autophagy induced by varicella-zoster virus (VZV), a human herpesvirus. Although closely related to herpes simplex virus type 1 (HSV-1), VZV lacks the inhibitors of autophagy harbored within the HSV genome (1-3). Therefore, autophagy is abundant after VZV infection (4-6). Many of our studies have relied on autophagosome quantitation to gauge the level of autophagy under varying conditions of infection (7). For these assays, we have enumerated autophagosomes (puncta) in the cytoplasm after immunolabeling with commercial LC3 antibody reagents (7). Depending on the conditions, we occasionally noticed what appeared to be puncta in the nuclei of the infected cells. In two recent autophagy articles $(8,9)$, other investigators had identified LC3-positive puncta in the nuclei of their stressed cells. They have implied that these LC3-positive puncta may be related to autophagy. Based on our extensive observations investigating VZV-induced autophagy, we postulate that these nuclear puncta are not related to autophagosome production but instead are related to the antibody reagent and other experimental conditions under which the microscopy experiment is carried out.

Based on prior autophagy experiments in our laboratory, we first postulated that different anti-LC3 antibodies led to different levels of nuclear LC3 staining. The panels in Figure 1 illustrate the main differences between using a rabbit monoclonal antibody (\#2057-1; Epitomics, Cambridge, MA) versus a rabbit polyclonal antibody (\#Sc-28266; Santa Cruz, Biotechnology, Inc., Dallas, TX) in different cell lines. In immortalized human keratinocytes (TERT-HFK), both uninfected and VZV-infected cells were labeled with anti-LC3 antibodies, but there was a much greater amount of nuclear LC3 staining with the rabbit polyclonal anti-LC3 reagent (panels A and B). Panel D demonstrates that immunoreactivity (red) to VZV IE62, an abundant viral protein, was present in panel $\mathrm{B}$.
In Figure 1, panels $\mathrm{E}$ and $\mathrm{F}$ show similar results in melanoma cells (4). Panel E shows syncytia cytopathology induced by VZV infection. Interestingly, nuclei (blue color) within the syncytia did not show LC3 staining after immunolabeling with the rabbit monoclonal antibody (panel E), although cytoplasmic puncta were easily seen. In contrast, immunolabeling with the rabbit polyclonal antibody caused prominent nuclear LC3 staining, as noted by the green puncta within the blue nuclei (panel F). Similar nuclear LC3 patterns were also seen in infected MRC-5 fibroblasts after immunolabeling with a polyclonal antibody (not shown). In short, nuclear puncta were more easily seen with polyclonal anti-LC3 antibodies, regardless of the infected cell substrate.

We next postulated that the conditions for permeabilization of the cells before immunolabeling were very important in determining the level of nuclear LC3 staining. All of the studies cited above used Triton X-100 for permeabilization. In previous experiments, we have used Triton concentrations ranging from $0.02 \%$ to $0.1 \%$ for $1 \mathrm{~h}$ at room temperature $(R T)$, to permeabilize cells before immunolabeling with anti-LC3 antibody. In the experiment shown in Figure 1, panels $\mathrm{G}-\mathrm{I}$, infected cells were fixed with $2 \%$ paraformaldehyde and permeabilized with $0.02 \%(G), 0.05 \%(H)$, or $0.1 \%(H)$ Triton $\mathrm{X}-100$ and then immunolabeled with the rabbit polyclonal antibody against LC3. As the amount of Triton X-100 was increased, more nuclei contained LC3 staining. Note in particular that almost every blue nucleus in panel I contained green puncta. This effect has been observed in many different experiments in this laboratory using different cell types and conditions of infection. We also observed that some nuclear puncta were larger than typically seen in the cytoplasm of VZV-infected cells; whereas true cytoplasmic puncta are $590 \mathrm{~nm} \pm 240 \mathrm{~nm}$ (10), some nuclear puncta were greater than $1000 \mathrm{~nm}$ in diameter.

After observing these differences between cytoplasmic and nuclear puncta, we postulated that the nuclear puncta were not typical double-membraned autophago- somes. Because our laboratory has used transmission electron microscopy (TEM) to examine virus-infected cells for many years, we have a large archive of micrographs. We have already documented cytoplasmic autophagosomes by TEM in an earlier paper (5). For this report, we reexamined more than 70 micrographs to look for any structures that resembled autophagosomes within the nuclei of uninfected or infected cells (Figure 2). In virus-infected monolayers during later time points (11), many viral capsids were seen in the nuclei; capsids measure 75-100 nm in diameter (5). Because autophagosomes typically are 4to 6-fold larger, we should be able to easily identify these structures, if they were present in the nuclei of these cells (Figure 2, E-H). Note the diameter of a virion in a cytoplasmic vacuole as another size control (Figure 2, circled in panel H). However, we found no double-membraned structures within nuclei (Figure 2). We also examined nuclear preparations from uninfected MRC-5 fibroblasts for the presence of structures that resembled autophagosomes. As seen in representative TEM images in Figure 2, A-D, no double-membraned structures were found in uninfected nuclei. Therefore, punctate LC3 staining within nuclei of cells was not attributable to the LC3-II embedded in the membranes of autophagosomes or organelles resembling autophagosomes.

We were intrigued when we read two recent autophagy papers in which the authors had observed what were called nuclear puncta identified by various anti-LC3 antibody reagents $(8,9)$. The authors were uncertain as to their function but speculated that the nuclear puncta may be related to autophagy. We know of no reason why our data about VZV-induced autophagy should not be applicable broadly. In particular, viral capsids in the nucleus provide a valuable marker for any structures $>100 \mathrm{~nm}$. Yet we see no structures compatible in size with autophagosomes in nuclei. Based on data acquired in our VZV-induced autophagy system, therefore, we postulate that these nuclear puncta are not related to autophagosome production. 
Figure 1. Effects of antibody choice and permeabilization on nuclear LC3 immunoreactivity. (A-D) TERTHFK cells, uninfected ( $A$ and $C$ ) or virus infected ( $B$ and D), were permeabilized with $0.05 \%$ Triton X-100 and labeled with Epitomics rabbit monoclonal antiLC3 (Epi LC3; panel A) or Santa Cruz rabbit polyclonal anti-LC3 (SC LC3; panel B) (both green), MAb 5C6 against VZV IE62 protein (red) (panel D), and the blue fluorescent H33342 DNA stain (Invitrogen) (panels A-D). Infections and immunolabeling were performed as described (6). Briefly, cells were grown on glass coverslips in 6-well dishes. After infection, monolayers were fixed, permeabilized, and blocked in 5\% nonfat milk with $2.5 \%$ normal goat serum in PBS for 2 h. Cells were then immunolabeled (primary antibody overnight at $4^{\circ} \mathrm{C}$; secondary antibody for $2 \mathrm{~h}$ at RT). Coverslips were mounted on glass slides and viewed on a Zeiss 710 Laser Scanning Confocal Microscope (Zeiss, Pleasanton, CA). Images were analyzed using Zen 2009 (Zeiss) software. LC3 puncta were seen in the cytoplasm of TERT-HFK cells when labeled with a rabbit monoclonal antibody against LC3 (A), but a rabbit polyclonal antibody against LC3 labeled mostly puncta in the nucleus, with some cytoplasmic staining (B). ( $E$ and F) Infected melanoma cells were permeabilized with $0.05 \%$ Triton X-100 and labeled with DNA stain (blue) and either Epitomics rabbit monoclonal anti-LC3 (Epi LC3; panel E) or Santa Cruz rabbit polyclonal anti-LC3 (SC LC3; panel F). In these cells, the monoclonal antibody labeled mainly cytoplasmic LC3 (E), but the polyclonal antibody stained primarily nuclear LC3 (F). (G-I) Three infected melanoma monolayers were fixed and permeabilized with increasing amounts of Triton X-100 - 0.02\% (G), $0.05 \%(\mathrm{H})$, or $0.1 \%(\mathrm{I})$ - and then labeled with a rabbit polyclonal antibody to LC3 (green) and the DNA stain (blue). Although occasional nuclei showed some LC3 staining at the $0.02 \%$ Triton X-100 concentration, nuclear LC3 staining increased greatly from $0.05 \%$ to $0.1 \%$. All images are shown are at a final magnification of $630 \times$, and all scale bars represent $20 \mu \mathrm{m}$.
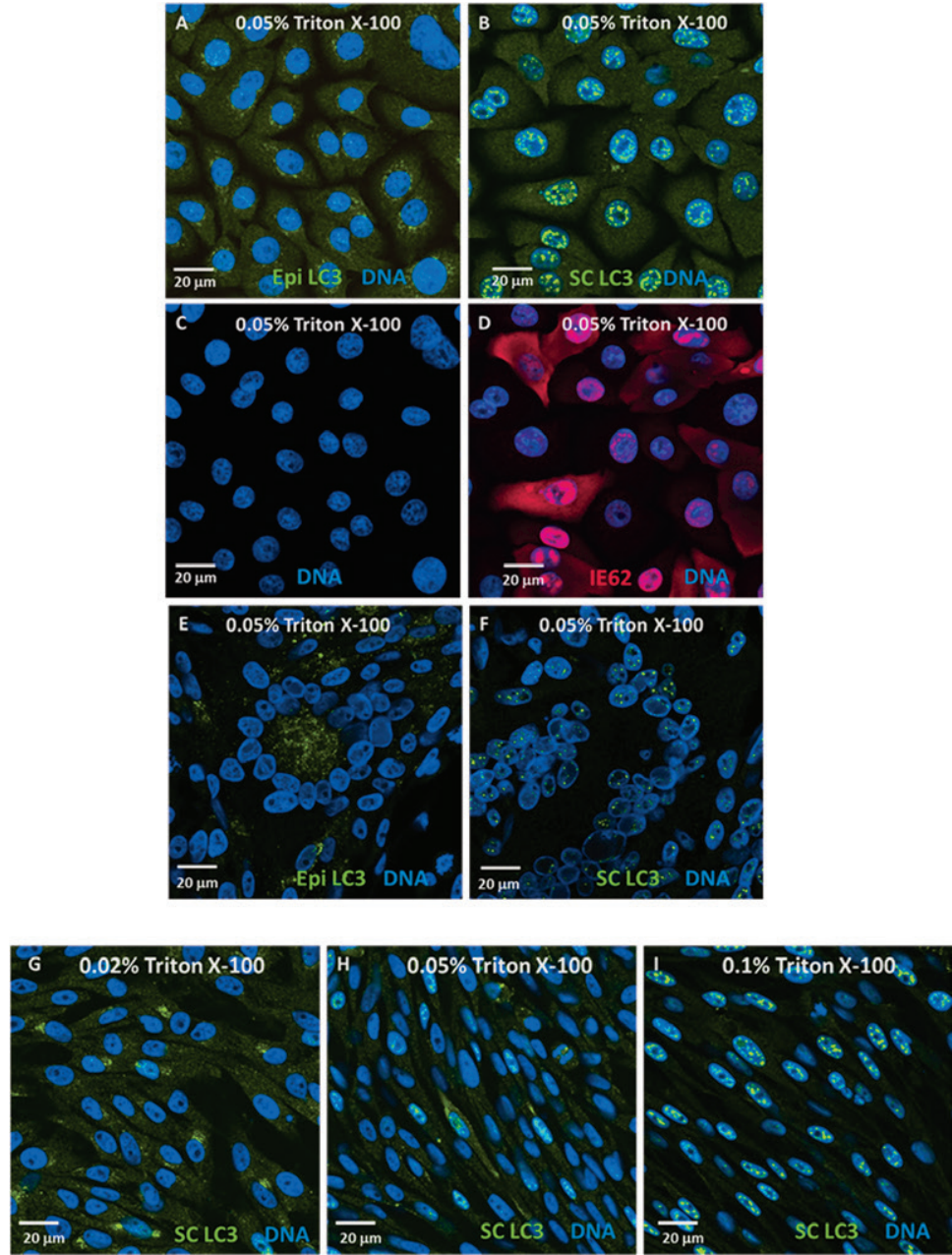
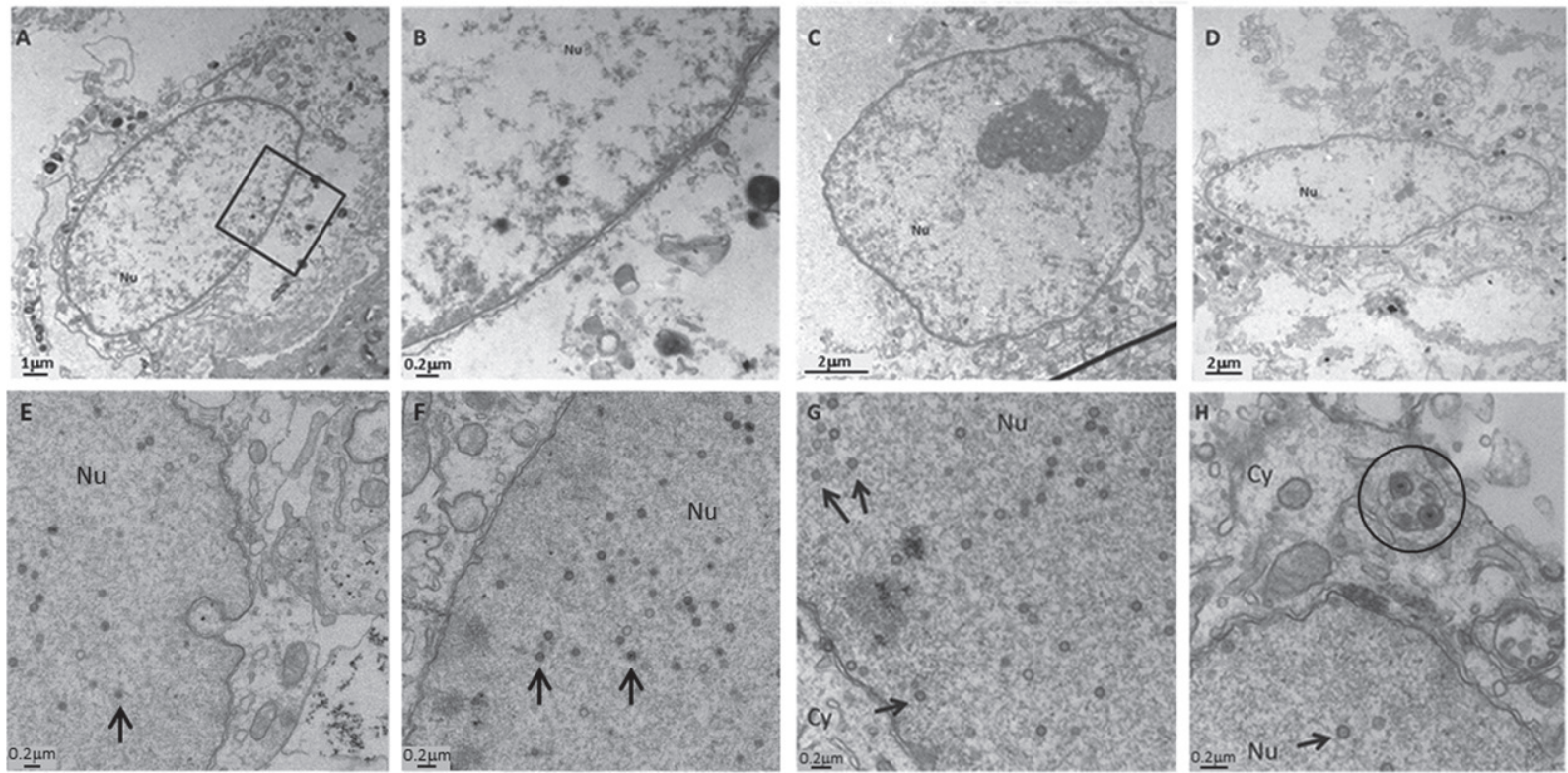

Figure 2. Absence of double-membraned autophagosomal structures in electron micrographs of nuclei of uninfected and virus-infected cells. (A-D) Nuclei isolated from uninfected cells showed no double-membraned structures resembling autophagosomes when examined by transmission electron microscopy (TEM). (B) Magnification of the region within the black box in panel A. (E-H) Melanoma cells infected with VZV showed no autophagosomal-like structures in the nucleus. Cells were infected for $24 \mathrm{~h}(\mathrm{E})$ or $72 \mathrm{~h}(\mathrm{~F}-\mathrm{H})$ and imaged by TEM. Black arrows within nuclei point to viral capsids approximately $75-$ $100 \mathrm{~nm}$ in diameter. The circle in $(\mathrm{H})$ encloses virions within a cytoplasmic vacuole. $\mathrm{Cy}=$ cytoplasm; $\mathrm{Nu}=$ nucleus. Scale bars are included in each panel. 


\section{SIXTH EDITION}

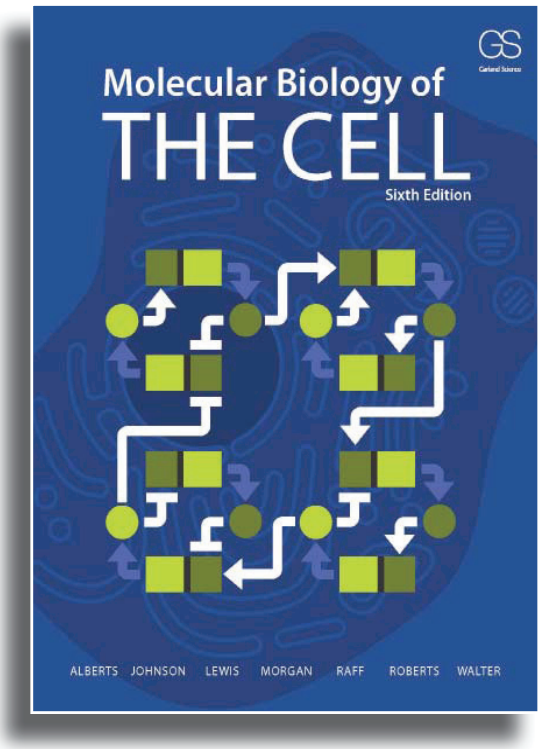

ALBERTS • JOHNSON

LEWIS・MORGAN • RAFF ROBERTS • WALTER

\section{December 2014}

$1,464 \mathrm{pp} \cdot 1,492$ illus

Hardback • 978-0-8153-4432-2

$A^{s}$ $s$ the amount of information in biology expands dramatically, it becomes increasingly important for textbooks to distill the vast amount of scientific knowledge into concise principles and enduring concepts. As with previous editions, Molecular Biology of the Cell, Sixth Edition accomplishes this goal with clear writing and beautiful illustrations. The Sixth Edition has been extensively revised and updated with the latest research in the field of cell biology, and it provides an exceptional framework for teaching and learning.

\section{garlandscience.com/mboc6}

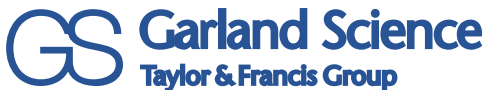

Instead, we conclude that a likely explanation for nuclear puncta is the formation of LC3 aggregates within the nuclei and their detection by the primary anti-LC3 antibody reagent (12). LC3-II protein is easily detectable in the nucleus (13). Earlier studies of direct precipitation of antigen by antibody by this laboratory and others demonstrate that aggregates are readily bound by polyclonal antisera (14). Furthermore, aggregate detection is more pronounced with polyclonal as opposed to monoclonal antibody reagents. In addition, the nonionic detergent Triton X-100 does not hinder the detection of antigen-antibody aggregates at the concentrations used for permeabilization (15).

Finally, we note that we are not advising that polyclonal anti-LC3 antibody reagents be avoided in autophagy studies. We are instead pointing out the optimal experimental conditions for their use in the detection of cytoplasmic puncta by confocal microscopy techniques.

\section{Author contributions}

E.M.B. designed experiments, obtained and analyzed the data, and prepared the manuscript. J.E.C. and W.J. contributed to the study design, obtained and analyzed the data, and contributed to manuscript preparation. C.G. supervised the study and prepared the manuscript.

\section{Acknowledgments}

We thank A. Klingelhutz (University of lowa) for his gift of the TERT-HFK cells. This autophagy research was supported by National Institutes of Health $(\mathrm{NIH})$ grant Al89716 (C.G.). The University of lowa Central Microscopy Research Facility is supported by NIH grant 1S10RR025439. This paper is subject to the NIH Public Access Policy.

\section{Competing interests}

The authors declare no competing interests.

\section{References}

1. Gobeil, P.A. and D.A. Leib. 2012. Herpes simplex virus gamma 34.5 interferes with autophagosome maturation and antigen presentation in dendritic cells. MBio. 3:e00267-12.

2. Davison, A.J. and J.E. Scott. 1986. The complete DNA sequence of varicella-zoster virus. J. Gen. Virol. 67:1759-1816.

3. Orvedahl, A., D. Alexander, Z. Talloczy, Q. Sun, Y. Wei, W. Zhang, D. Burns, D.A. Leib, and B. Levine. 2007. HSV-1 ICP34.5 confers neurovirulence by targeting the Beclin 1 autophagy protein. Cell Host Microbe. 1:23-35.
4. Carpenter, J.E., W. Jackson, L. Benetti, and C. Grose. 2011. Autophagosome formation during varicella-zoster virus infection following endoplasmic reticulum stress and the unfolded protein response. J. Virol. 85:9414-9424.

5. Takahashi, M.N., W. Jackson, D.T. Laird, T.D. Culp, C. Grose, J.I. Haynes 2nd, and L. Benetti. 2009. Varicella-zoster virus infection induces autophagy in both cultured cells and human skin vesicles. J. Virol. 83:5466-5476.

6. Buckingham, E.M., J.E. Carpenter, W. Jackson, and C. Grose. 2014. Autophagy and the effects of its inhibition on varicella-zoster virus glycoprotein biosynthesis and infectivity. J. Virol. 88:890-902.

7. Klionsky, D.J., F.C. Abdalla, H. Abeliovich, R.T. Abraham, A. Acevedo-Arozena, K. Adeli, L. Agholme, M. Agnello, et al. 2012. Guidelines for the use and interpretation of assays for monitoring autophagy. Autophagy. 8:445-544.

8. Martinez-Lopez, N., D. Athonvarangkul, P. Mishall, S. Sahu, and R. Singh. 2013. Autophagy proteins regulate ERK phosphorylation. Nat. Commun. 4:2799.

9. Corum, D.G., P.N. Tsichlis, and R.C. MuiseHelmericks. 2014. AKT3 controls mitochondrial biogenesis and autophagy via regulation of the major nuclear export protein CRM-1. FASEB J. 28:395-407.

10. Jackson, W., M. Yamada, T. Moninger, and C. Grose. 2013. Visualization and quantitation of abundant macroautophagy in virus-infected cells by confocal three-dimensional fluorescence imaging. J. Virol. Methods. 193:244-250.

11. Hutchinson, J.A. 2007. Thesis: Imaging analysis of varicella-zoster virus infection with emphasis on glycoprotein C, p. 224. Interdisciplinary Studies. University of lowa, lowa City, IA.

12. Kuma, A., M. Matsui, and N. Mizushima. 2007. LC3, an autophagosome marker, can be incorporated into protein aggregates independent of autophagy: caution in the interpretation of LC3 localization. Autophagy. 3:323-328.

13. Karim, M.R., T. Kanazawa, Y. Daigaku, S. Fujimura, G. Miotto, and M. Kadowaki. 2007. Cytosolic LC3 ratio as a sensitive index of macroautophagy in isolated rat hepatocytes and $\mathrm{H} 4-\mathrm{II}-\mathrm{E}$ cells. Autophagy. 3:553-560.

14. Horwitz, M.S. and M.D. Scharff. 1969. Immunological precipitation of radioactively labeled viral proteins, p. 297-315. In K. Habel and N.P. Salzman (Ed.), Fundamental Techniques in Virology. Academic Press, New York, NY.

15. Qualtiere, L.F., A.G. Anderson, and P. Meyers. 1977. Effects of ionic and nonionic detergents on antigen-antibody reactions. J. Immunol. 119:16451651.

Received 20 August 2014; accepted 29 September 2014.

BioTechniques 57:241-244 (November 2014) doi 10.2144/000114226

Erin M. Buckingham, John E. Carpenter, Wallen Jackson, and Charles Grose

Virology Laboratory, University of lowa Children's Hospital, lowa City, IA

Address correspondence to Charles Grose, Virology Laboratory, University of lowa Children's Hospital, lowa City, IA. E-mail: charles-grose@uiowa.edu

To purchase reprints of this article, contact: biotechniques@fosterprinting.com 